\section{Limited Generalizability of a Retrospective Chart Review Comparing 3 Recombinant FVIII Products for Hemophilia A Prophylaxis}

We have read with great interest the April issue of JMCP, which featured an article by Simpson et al. that was a retrospective chart review comparing bleeding rates and factor consumption of 3 recombinant factor VIII products for hemophilia A prophylaxis. ${ }^{1}$ We commend the authors for conducting this timely analysis, given the increased number of new therapies that have become available to the hemophilia community.

In the introduction, the authors discuss the concepts and definitions of long-acting, standard half-life (SHL), and extended half-life (EHL) factor therapy. To provide a framework for future assessments of rFVIII products, Mahlangu et al. (2018) published an evidence-based definition for EHL rFVIII. ${ }^{2}$ Based on 3 criteria defined to classify an rFVIII as an EHL, it should be noted that rFVIII-SingleChain does not meet the criteria as an EHL, by definition and design, ${ }^{2}$ as defined by Lambert et al. (2018). ${ }^{3}$

Also, we highlight additional limitations, beyond those listed in the Simpson et al. article, that potentially skews study results and interpretation.

First, since the matching methodology is not documented in the article (e.g., 1:1 match, propensity score matching), it is challenging to understand the relevance of the findings. For example, while the study attempted to match cohorts on age and severity, the rVIII-SingleChain cohort has a much lower mean weight versus the rFVIIIFc $(-12 \mathrm{~kg})$ and PEG-rFVIII $(-22 \mathrm{~kg})$ cohorts. While there was an attempt to account for weight differences as part of the ANCOVA and normalization of factor utilization to IU per kg per week, given its significant effect on consumption, the analysis would be strengthened if the matching variables included weight or additional sensitivity analysis conducted.

Second, other known confounders of treatment outcomes of hemophilia were not accounted for in the analysis. Previous treatment regimen, previous bleeding events, existence of arthropathy, inhibitor history, and treatment center are examples of potential confounders that should be addressed. ${ }^{4,5}$

Third, 17 (42.5\%) of rFVIIIFc patients had a weekly dosing frequency other than 2 or 3 times per week, with 14 (35\%) dosing $<2$ times per week ( $\mathrm{n}=8$ [20\%] on weekly dosing). Based on Table 3 in the article, it can be calculated that the mean weekly factor consumption for these 17 patients was $77.6 \mathrm{IU}$ per $\mathrm{kg}$. It should be noted that $10 \%$ of the rFVIII-SingleChain group and none of the PEG-rFVIII group were on a weekly dosing schedule. Therefore, the comparison in Table 3 would be subject to bias; it would be of interest to assess outcomes in comparable dosing strata.

Fourth, the article limits the pre- vs. post-switch subgroup analysis to the rVIII-SingleChain cohort only; inclusion of all 3 cohorts in the switch analysis would have strengthened the study as a comprehensive comparative analysis.
In summary, the results reported by Simpson et al. should be interpreted with caution. The generalizability of the results may be under question because of methodological considerations concerning ambiguity around the method for matching. In addition, the robustness of the analysis could be strengthened if the authors did not limit themselves to a switch subgroup analysis for the rVIII-SingleChain cohort only.

\section{Ronald Preblick, PharmD, MPH}

Sanofi Genzyme, Cambridge, MA

Ronald.Preblick@sanofi.com

\section{Cori Gray, PharmD \\ Sanofi Genzyme, Cambridge, MA}

\section{Talaha Ali, MD}

Sanofi Genzyme, Cambridge, MA

Pronabesh DasMahapatra, MD, MPH

Sanofi Genzyme, Cambridge, MA

\section{DISCLOSURES}

No funding supported the writing of this letter. Preblick, Ali, and DasMahapatra are employees and shareholders of Sanofi Genzyme. Gray is a postdoctoral fellow at Sanofi Genzyme and Rutgers University.

\section{REFERENCES}

1. Simpson ML, Desai V, Maro GS, Yan S. Comparing factor use and bleed rates in U.S. hemophilia A patients receiving prophylaxis with 3 different long-acting recombinant factor VIII products. J Manag Care Spec Pharm. 2020;26(4):504-12.

2. Mahlangu J, Young G, Hermans C, et al. Defining extended half-life rFVIII-a critical review of the evidence. Haemophilia. 2018;24(3):348-58

3. Lambert T, Benson G, Dolan G, et al. Practical aspects of extended half-life products for the treatment of haemophilia. Ther Adv Hematol. 2018;9(9):295-308.

4. Bladen M, Main E, Hubert N, et al. Factors affecting the Haemophilia Joint Health Score in children with severe haemophilia. Haemophilia. 2013;19(4):626-31.

5. Oldenburg J, Hay CRM, Jimenez-Yuste V, et al. Design of a prospective observational study on the effectiveness and real-world usage of recombinant factor VIII Fc ( $\mathrm{rFVIIIFc)}$ compared with conventional products in haemophilia A: the A-SURE study. BMJ Open. 2019;9:3028012.

\section{The Authors Respond}

We thank Preblick et al. for their comments on our article. rVIII-SingleChain has been developed with an improved pharmacokinetic and pharmacodynamic profile compared with standard-acting recombinant FVIII (rFVIII) products ${ }^{1}$; this allows for a less frequent dosing regimen compared with standard-acting rFVIII, similar to regimens achievable with other long-acting products. Long-acting products are generally considered to include all the newer rFVIII products with 